OPEN ACCESS

Edited by:

Liang Li,

Tsinghua University, China

Reviewed by:

Stefan Sawall,

German Cancer Research Center

(DKFZ), Germany

Yunsong Zhao,

Capital Normal University, China

*Correspondence:

Zhe Wang

wangzhe@ihep.ac.cn

Specialty section:

This article was submitted to

Medical Physics and Imaging,

a section of the journal

Frontiers in Physics

Received: 01 September 2020

Accepted: 30 November 2020

Published: 08 February 2021

Citation:

Zhang Z, Zhang X, Hu J, Xu Q, Li M, Wei C, Wei $L$ and Wang $Z$ (2021) An Optimized K-Edge Signal Extraction Method for K-Edge Decomposition Imaging Using a Photon Counting Detector.

Front. Phys. 8:601623.

doi: 10.3389/fphy.2020.601623

\section{An Optimized K-Edge Signal Extraction Method for K-Edge Decomposition Imaging Using a Photon Counting Detector}

\author{
Zhidu Zhang ${ }^{1,2}$, Xiaomei Zhang ${ }^{1,2}$, Jinming $\mathrm{Hu}^{1,2}$, Qiong $\mathrm{Xu}^{1,3}$, Mohan $\mathrm{Li}^{1,3}$, Cunfeng Wei ${ }^{1,2,3}$ \\ Long Wei ${ }^{1,2,3}$ and Zhe Wang ${ }^{1,3 *}$
}

${ }^{1}$ Beijing Engineering Research Center of Radiographic Techniques and Equipment, Institute of High Energy Physics, Chinese Academy of Sciences, Beijing, China, ${ }^{2}$ School of Nuclear Science and Technology, University of Chinese Academy of Sciences, Beijing, China, ${ }^{3}$ Jinan Laboratory of Applied Nuclear Science, Jinan, China

In K-edge decomposition imaging for the multienergy system with the photon counting detectors (PCDs), the energy bins significantly affect the intensity of the extracted K-edge signal. Optimized energy bins can provide a better K-edge signal to improve the quality of the decomposition images and have the potential to reduce the amount of contrast agents. In this article, we present the Gaussian spectrum selection method (GSSM) for the multienergy K-edge decomposition imaging which can extract an optimized K-edge signal by optimizing energy bins compared with the conventional theoretical attenuation selection method (TASM). GSSM decides the width and locations of the energy bins using a simple but effective model of the imaging system, which takes the degraded energy resolution of the detector and the continuous $x$-ray spectrum into consideration. Besides, we establish the objective function, difference of attenuation to relative standard deviation ratio (DAR), to determine the optimal energy bins which maximize the K-edge signal. The results show that GSSM gets a better K-edge signal than TASM especially at the lower concentration level of contrast agents. The new method has the potential to improve the contrast and reduce the amount of contrast agents.

Keywords: multienergy imaging, photon counting detector, K-edge decomposition imaging, K-edge signal, energy bins

\section{INTRODUCTION}

K-edge decomposition imaging has remarkable potential in some clinical applications like the $\mathrm{x}$-ray oncology imaging for the breast and the abdomen $[1,2]$. At present, the K-edge decomposition imaging is commonly realized using traditional dual-energy devices $[3,4]$. There are four types of devices for dual-energy imaging: the sequence scan device, the dual-source device [5], the dual-layer detector device [6], and the fast $\mathrm{kVp}$ switching device [7]. The sequence scan device requires double exposures which increase the motion artifacts [8]. The dual-source device has a much more complex system and the images obtained have different phases. The dual-layer detector device can obtain images at the same phase in one exposure, but its energy resolution performance is relatively weak which goes against the quality of resulting images. The fast $\mathrm{kVp}$ switching device has a higher requirement of the imaging system and still has the problem of phase-matching. Besides, the energy mixing of the photons weakens the K-edge signal [9]. 


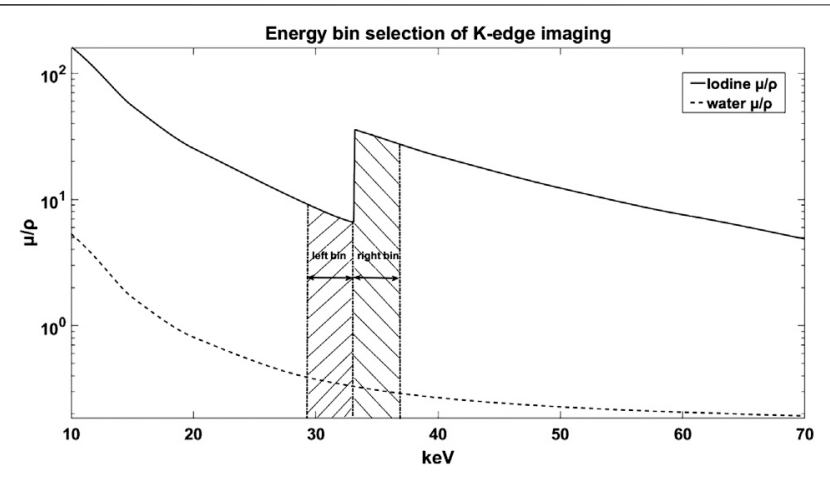

FIGURE 1 | The mass attenuation coefficient of water and iodine for $\mathrm{x}$-rays. The shadow parts represent the general energy bins selection for K-edge subtraction imaging.

In recent years, the development of the photon counting detectors (PCDs) attracted much attention for multienergy imaging [10]. Multienergy imaging based on PCDs can obtain images within different energy bins in one exposure and thereby can solve the exiting problems of the dual-energy imaging to a certain extent $[11,12]$. Also, because of the energy resolving ability and adjustable energy thresholds of PCDs, it can obtain more precise spectrum information to improve the K-edge signal [13]. Therefore, multienergy imaging based on PCDs is one of the research focuses.

The energy bins used for the K-edge decomposition imaging based on the PCDs significantly affect the contrast of the processed images [14, 15]. The energy bins decided by the conventional theoretical attenuation selection method (TASM) are widely used for K-edge decomposition imaging as shown in Figure 1. In this figure, the two energy bins are symmetrical on both sides of the theoretical K-edge position [16, 17], but the limited energy resolution of the PCDs distorts the attenuation curve [18] which will lead to the deviation of the energy bins in TASM. Several physical effects of the PCD are responsible for the degradation of energy resolution, including Compton scattering [19], charge sharing [20], pulse pileup [21], and fluorescence emission [22]. Moreover, the continuous x-ray spectrum also flattens the K-edge signal [23]. The weakened K-edge signal can further influence the quality of the decomposition image and increase the amount of the contrast agents.

Some authors optimized the K-edge decomposition algorithm in previous studies [24-26] to guarantee the results of the decomposed images. Mang Feng et al. [27] and Ding et al. [28] optimized the $\mathrm{x}$-ray spectrum which have the potential to get a better image contrast.

Another effective way to improve the quality of the K-edge decomposition imaging is optimizing the energy bins. Previous work was mostly focused on optimizing the width of the energy bins used for K-edge imaging, He et al. decided the energy bin width by signal difference to noise ratio (SDNR) next to the K-edge position [29]. Bo Meng et al. used the redescribed signal to noise ratio (SNR) to obtain the energy bin width next to the K-edge position [30]. Seung-Wan Lee et al. carried out some simulation work on optimizing the energy bins [31]. However, few studies considered the effects of the degraded energy resolution and the continuous $\mathrm{x}$-ray spectrum on energy bins optimization which distort the attenuation curve and lead to a deviation of the energy bins. To take these negative factors into consideration, Silvia Pani et al. selected the energy bins by mapping the spectrum passing through the contrast agents [25], but the disadvantage of this approach is being too tedious for the practical application.

In this work, we propose the Gaussian spectrum selection method (GSSM) for multienergy imaging to increase the intensity of K-edge signal. It takes the degraded energy resolution and the continuous $\mathrm{x}$-ray spectrum into consideration by modeling the imaging system and decides the optimal energy bins by the objective function, difference of attenuation to relative standard deviation ratio (DAR), proposed in this research. GSSM can obtain both the width and the locations of the optimized energy bins without the spectrum mapping process. The experimental results in Experimental Materials and Designs show that the decomposition image obtained by the GSSM has a higher quality than that obtained by the TASM.

\section{METHOD}

The method of this study to optimize the energy bins is based on the modeling of the multienergy imaging system. We estimate the influence of the energy resolution $\left(R_{E}\right)$ with the Gaussian convolution to the theoretical mass attenuation curve and estimate the influence of the continuous $\mathrm{x}$-ray spectrum distribution by calculating the equivalent mass attenuation coefficients of the energy bins. Finally, we establish the objective function DAR to determine the optimal energy bins which maximize the K-edge signal.

\section{Imaging System Modeling}

The mass theoretical attenuation curve of the material with K-edge changes to the shape shown in Figure 2 under the influence of $R_{E}$; this can be estimated using Gaussian convolution $[18,32]$. The theoretical mass attenuation

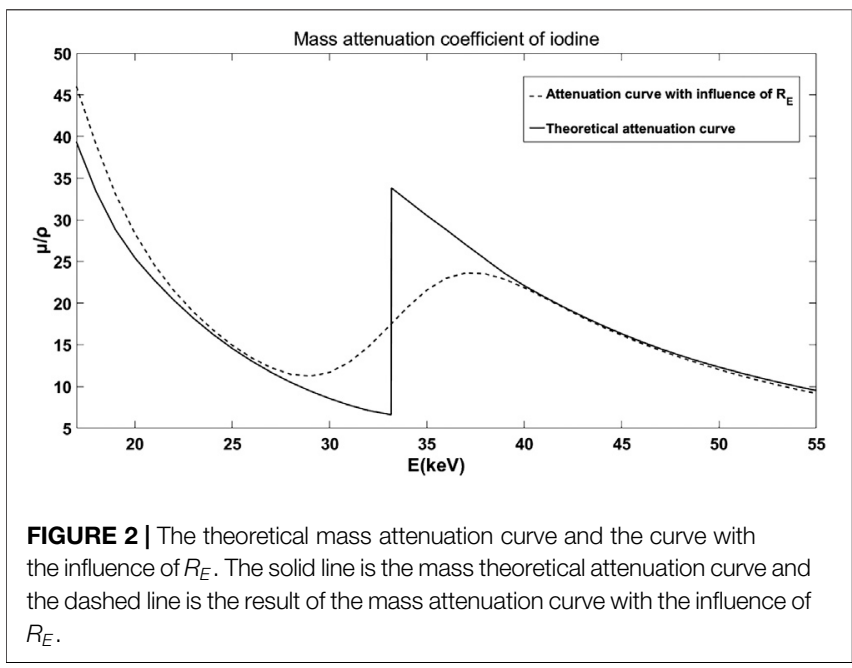




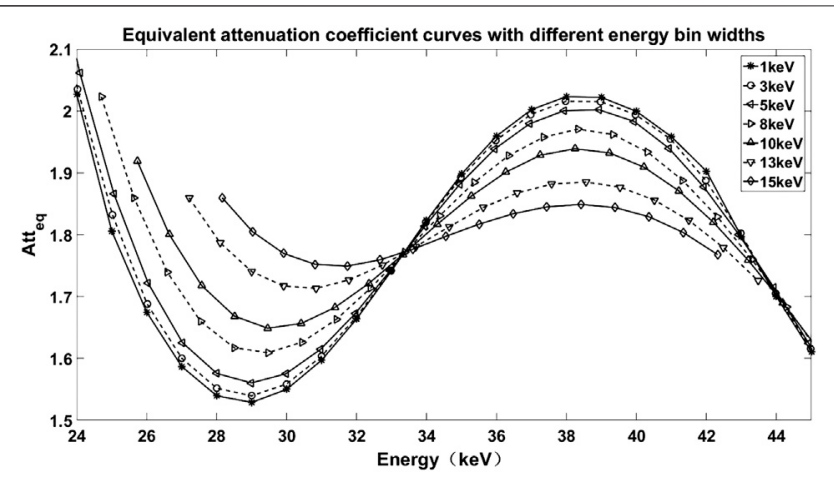

FIGURE 3 | Equivalent mass attenuation coefficient curves of iodine with different energy bin widths.

coefficient data is acquired from the National Institute of Standards and Technology (NIST) [33] and is recorded as $A t t_{0}(E)$. The spectrum of the $\mathrm{x}$-ray tube, $P_{0}(E)$, is estimated by the simulation software SpekCalc [34, 35]. The process of Gaussian convolution is described in

$$
\begin{aligned}
A t t_{G}(E) & =A t t_{0}(E) \otimes C(E), \\
P_{G}(E) & =P_{0}(E) \otimes C(E),
\end{aligned}
$$

where

$$
\begin{gathered}
C(E)=\frac{1}{\sqrt{2 \pi \sigma_{E}^{2}}} \exp \left(-\frac{E^{2}}{2 \sigma_{E}^{2}}\right), \\
\sigma_{E}=\frac{R_{E}}{2.355}=\frac{R_{0} \cdot E_{K} \cdot \sqrt{\frac{E_{0}}{E_{K}}}}{2.355}
\end{gathered}
$$

$\operatorname{Att}_{G}(E)$ is the mass attenuation curve after Gaussian convolution and $P_{G}(E)$ is the spectrum after Gaussian convolution. $C(E)$ is the Gaussian convolution kernel at the corresponding energy, which is determined by $R_{E}$. $C(E)$ has a complex form with energy on the overall mass attenuation curve, but at the K-edge position $\left(E_{K}\right)$ it can be calculated as in (2). The $R_{0}$ in (2) is the energy resolution at the known energy position $E_{0}$, and the $E_{K}$ is the $\mathrm{K}$-edge energy position of the material.

The influence of spectrum is expressed by the equivalent mass attenuation coefficient $\left(A t t_{e q}(B)\right)$ of the energy bin in (3) [36, 37], where $B$ represents the corresponding energy bin:

$$
A t t_{e q}(B)=\frac{\int_{B} A t t_{G}(E) P_{G}(E) d E}{\int_{B} P_{G}(E) d E}
$$

\section{Objective Function DAR}

The quality of the decomposition image is determined by not only the contrast but also the noise level.

This research proposes the objective function, the difference of attenuation to relative standard deviation ratio (DAR) to maximize the K-edge signal, and the meaning of DAR is shown in

$$
D A R=\frac{\Delta A t t_{e q}}{N}
$$

where $\Delta A t t_{e q}$ represents the difference of equivalent mass attenuation coefficients between the left and the right energy bins of the K-edge. $N$ represents the total noise level of these two images. They are mutually restrictive: a wide bin can reduce the noise level while weakening $\Delta A t t_{e q}$ (shown in Figure 3). Conversely, a narrow bin can maintain the $\Delta A t t_{e q}$ while increasing the noise level of the image.

The detailed derivation of DAR is provided in Appendix 1. The final DAR expression is shown in

$$
\begin{aligned}
& \frac{\operatorname{DAR}\left(B^{L}, B^{R}\right)}{\sqrt{\frac{\left(A t t_{e q}\left(B^{R}\right) *(\rho d)-A t t_{e q}\left(B^{L}\right) *(\rho d)\right)^{2}}{\left(\int_{B^{L}} P_{G}(E) d E\right) \mathrm{e}^{-A t t e q}\left(B^{L}\right) *(\rho d)}+\frac{1}{\left(\int_{B^{R}} P_{G}(E) d E\right) \mathrm{e}^{-A t t e q}\left(B^{R}\right) *(\rho d)}}}
\end{aligned}
$$

where $(\rho d)$ is the mass thickness of the sample and $B^{L}$ and $B^{R}$ are the left and the right energy bins of K-edge. $A t t_{e q}\left(B^{\mathrm{L}}\right)$ and $A t t_{e q}\left(B^{\mathrm{R}}\right)$ are the equivalent mass attenuation coefficients of $B^{L}$ and $B^{R}$, respectively.

The optimal solution $\widetilde{B^{L}}$ and $\widetilde{B^{R}}$ is expressed in (6). Since the difference of equivalent mass attenuation coefficients should be large enough in the contrast agents while it should be small enough in the background between the two energy bins, the solution space of the optimal energy bins is limited to a very small area:

$$
\begin{aligned}
& \max \operatorname{DAR}\left(\overline{B^{L}}, \overline{B^{R}}\right), \\
& \overline{B^{L}} \in\left[E_{K}-n \sigma_{E}, E_{K}\right], \\
& \overline{B^{R}} \in\left[E_{K}, E_{K}+n \sigma_{E}\right],
\end{aligned}
$$

where $E_{K}$ is the energy at the K-edge position and $\mathrm{n}$ is an empirical constant and $\overline{B^{L}}$ and $\overline{B^{R}}$ represent the mid-values of $B^{L}$ and $B^{R}$. In general, the optimal solution of (6) should be determined by analyzing the stationary points and the He ssian matrix of DAR. However, the analytic expressions of the derivatives for the DAR used in this research are difficult to get. Since the solution space is limited around the K-edge position, all energy bins in the solution space are calculated to determine the optimal solutions, which are corresponding to the maximum DAR.

Take iodine as an example, where the left bin and right bin images $S_{G}^{L}$ and $S_{G}^{R}$ acquired by GSSM can be expressed as follows:

$$
\left\{\begin{array}{l}
S_{G}^{L}=\left(\int_{\widetilde{B^{L}}} P_{G}(E) d E\right) \exp \left(-\left(A t t_{e q}\left(\widetilde{B^{L}}\right)_{I}(\rho d)_{I}+A t t_{e q}\left(\widetilde{B^{L}}\right)_{b g}(\rho d)_{b g}\right)\right) \\
S_{G}^{R}=\left(\int_{\widetilde{B^{R}}} P_{G}(E) d E\right) \exp \left(-\left(A t t_{e q}\left(\widetilde{B^{R}}\right)_{I}(\rho d)_{I}+A t t_{e q}\left(\widetilde{B^{R}}\right)_{b g}(\rho d)_{b g}\right)\right),
\end{array}\right.
$$




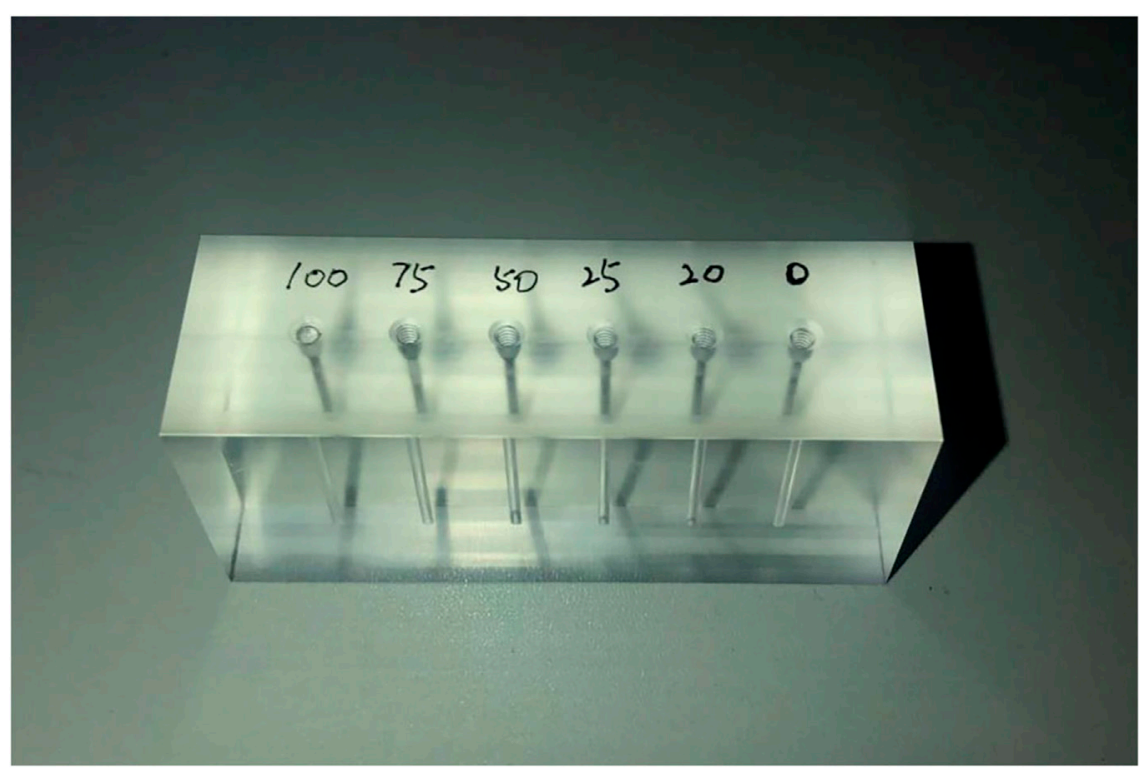

FIGURE 4 | Phantom used in experiments 3.3 and 3.4.

And the left bin and right bin images $S_{T}^{L}$ and $S_{T}^{R}$ acquired by TASM can be expressed as follows:

$$
\left\{\begin{array}{l}
S_{T}^{L}=\left(\int_{E_{K}-\omega}^{E_{K}} P_{0}(E) d E\right) \exp \left(-\left(\left(\frac{1}{\omega} \int_{E_{K}-\omega}^{E_{K}} A t t_{0}(E)_{I} d E\right)(\rho d)_{I}+\left(\frac{1}{\omega} \int_{E_{K}-\omega}^{E_{K}} A t t_{0}(E)_{b_{g}} d E\right)(\rho d)_{b_{g}}\right)\right) \\
S_{T}^{R}=\left(\int_{E_{K}}^{E_{K}+\omega} P_{0}(E) d E\right) \exp \left(-\left(\left(\frac{1}{\omega} \int_{E_{K}}^{E_{K}+\omega} A t t_{0}(E)_{I} d E\right)(\rho d)_{I}+\left(\frac{1}{\omega} \int_{E_{K}}^{E_{K}+\omega} A t t_{0}(E)_{b_{g}} d E\right)(\rho d)_{b_{g}}\right)\right)
\end{array}\right.
$$

where the $\omega$ represents the energy bin width. The different energy bins in the TASM and the GSSM lead to different final decomposition image qualities.

\section{Dual Energy Decomposition Algorithm}

We also take the iodine contrast agents as an example. Considering the superposition of background and iodine, the images for the left and the right energy bins next to the K-edge can be expressed as in (9) [25]:

$$
\left\{\begin{array}{l}
S^{L}=S_{0}^{L} \exp \left(-\left(\left(\frac{\mu}{\rho}\right)_{I}^{L}(\rho d)_{I}+\left(\frac{\mu}{\rho}\right)_{b g}^{L}(\rho d)_{b g}\right)\right) \\
S^{R}=S_{0}^{R} \exp \left(-\left(\left(\frac{\mu}{\rho}\right)_{I}^{\mathrm{R}}(\rho d)_{I}+\left(\frac{\mu}{\rho}\right)_{b g}^{R}(\rho d)_{b g}\right)\right)
\end{array}\right.
$$

where $\left(\frac{\mu}{\rho}\right)_{I}^{L},\left(\frac{\mu}{\rho}\right)_{I}^{\mathrm{R}}$ and $\left(\frac{\mu}{\rho}\right)_{b g}^{L},\left(\frac{\mu}{\rho}\right)_{b g}^{R}$ are the mass attenuation coefficients of iodine and background for the left and the right energy bins, $(\rho d)_{I}$ and $(\rho d)_{b g}$ are the mass thicknesses of iodine and background, and $S_{0}^{L}$ and $S_{0}^{R}$ are incident photon numbers, respectively. The attenuation characteristics A of these two energy bins can be expressed as follows:

$$
\left\{\begin{array}{l}
A^{L}=\ln \left(S_{0}^{L} / S^{L}\right)=\left(\frac{\mu}{\rho}\right)_{I}^{L}(\rho d)_{I}+\left(\frac{\mu}{\rho}\right)_{b g}^{L}(\rho d)_{b g} \\
A^{R}=\ln \left(S_{0}^{R} / S^{R}\right)=\left(\frac{\mu}{\rho}\right)_{I}^{\mathrm{R}}(\rho d)_{I}+\left(\frac{\mu}{\rho}\right)_{b g}^{R}(\rho d)_{b g}
\end{array}\right.
$$

The iodine information is calculated to get the iodineequivalent image with

$$
(\rho d)_{I}=\frac{\left(\frac{\mu}{\rho}\right)_{b g}^{L} A^{R}-\left(\frac{\mu}{\rho}\right)_{b g}^{R} A^{L}}{\left(\frac{\mu}{\rho}\right)_{b g}^{L}\left(\frac{\mu}{\rho}\right)_{I}^{R}-\left(\frac{\mu}{\rho}\right)_{b g}^{R}\left(\frac{\mu}{\rho}\right)_{I}^{L}}
$$

\section{EXPERIMENTAL MATERIALS AND DESIGNS}

\section{Imaging System}

The imaging system used in this research was a spectral microcomputed tomography (CT) prototype for small animals, which was independently developed by the Institute of High Energy Physics, Chinese Academy of Sciences. It can realize multienergy digital radiography (DR) and CT scanning by using the photon counting detector produced by XCounter. The sensor material of the detector is CdTe and its thickness was $0.75 \mathrm{~mm}$. The effective area was $153.6^{\star} 25.6 \mathrm{~mm}^{2}$. The detector has $1536 * 256$ pixels with a single pixel area of $100^{\star} 100 \mu \mathrm{m}^{2}$. It has two energy thresholds and it can detect the photons with the energy range from $10 \mathrm{keV}$ to $160 \mathrm{keV}$. The two energy thresholds determine the lower and higher boundary of an energy bin in one scanning. To acquire images with different 


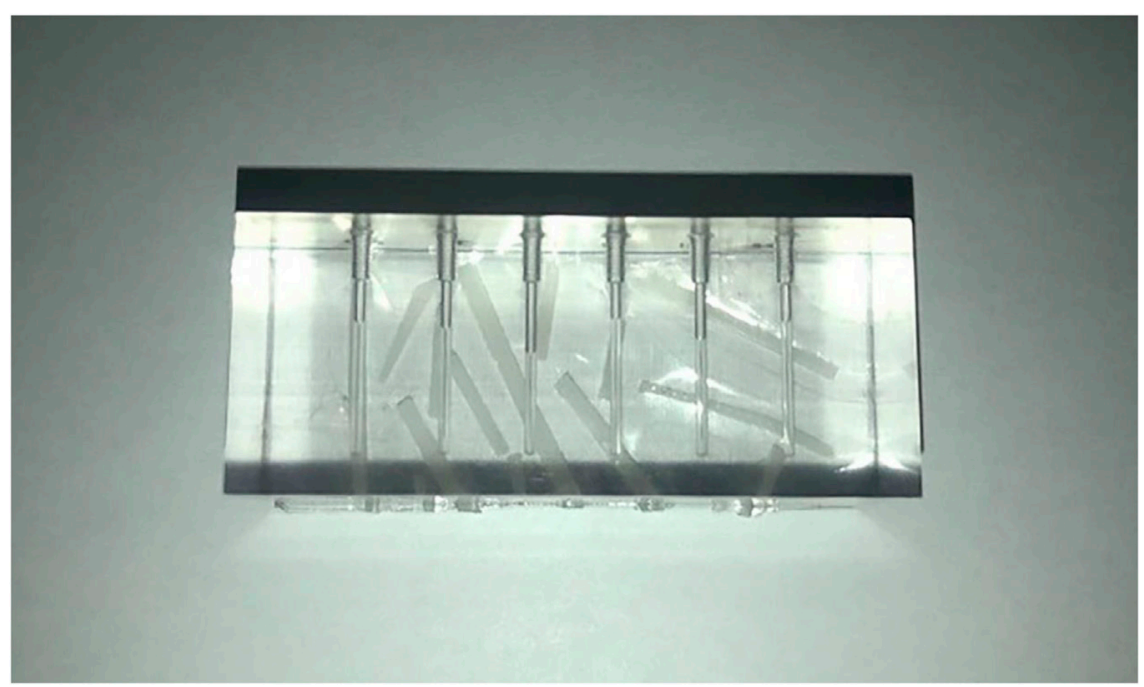

FIGURE 5 | Phantom used in the comparison experiment.
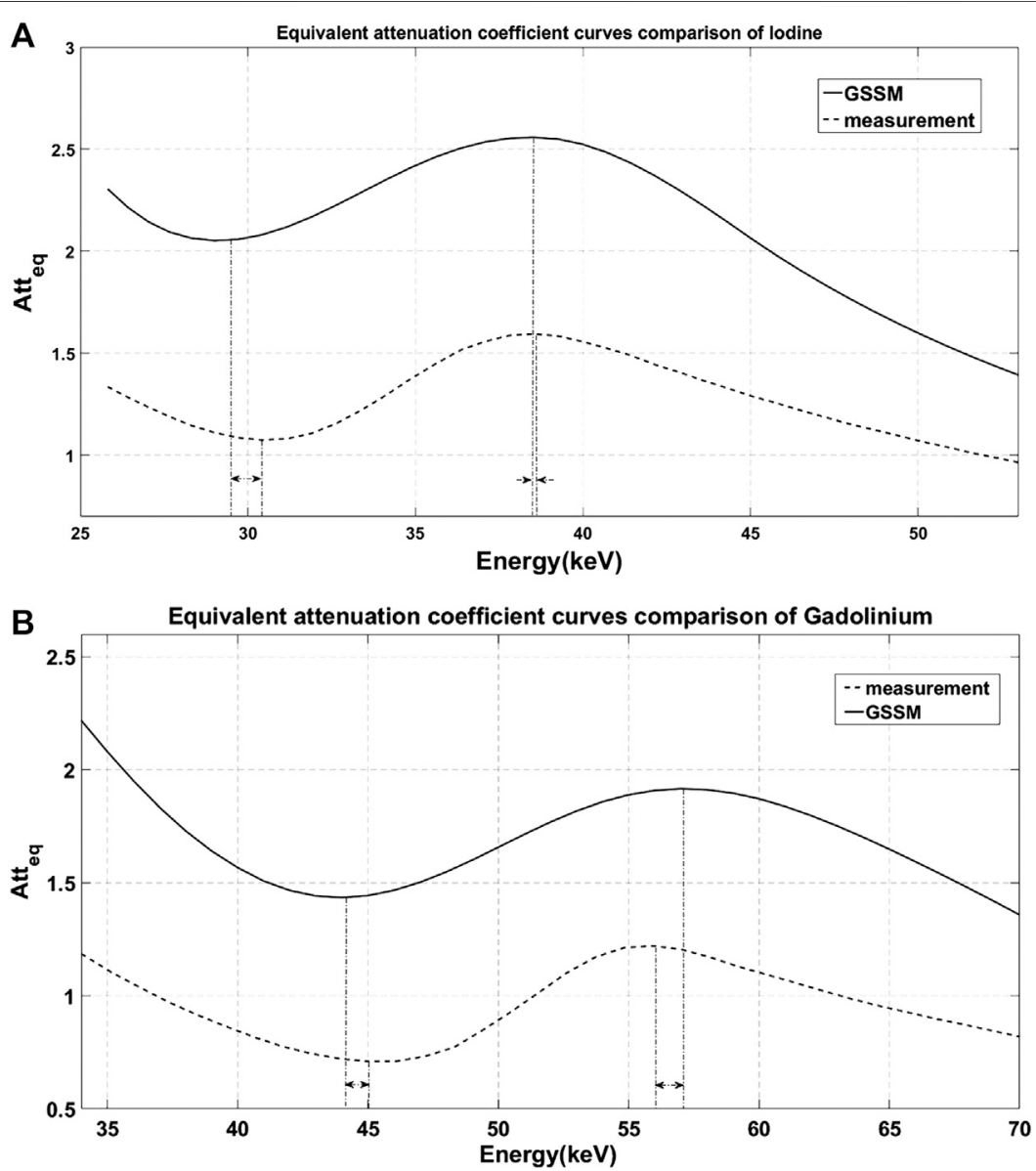

FIGURE 6 | Comparison of the equivalent attenuation coefficient curves obtained by GSSM (solid line) and measurement (dashed line). The dash-dotted lines represent the energy positions of the extreme points and the arrows show the errors between GSSM and measurement: (A) iodine results and (B) gadolinium results. 
TABLE 1 | The energy positions of the K-edge extreme points of iodine and gadolinium.

\begin{tabular}{|c|c|c|c|c|c|c|}
\hline \multirow{2}{*}{$\frac{\text { Material }}{\text { Method }}$} & \multicolumn{3}{|c|}{ lodine } & \multicolumn{3}{|c|}{ Gadolinium } \\
\hline & GSSM & Measurement & Error(\%) & GSSM & Measurement & Error(\%) \\
\hline Valley energy (keV) & 29.51 & 30.42 & 2.99 & 44.16 & 45.02 & 1.91 \\
\hline Peak energy(keV) & 38.54 & 38.61 & 0.18 & 57.1 & 56.15 & 1.69 \\
\hline
\end{tabular}

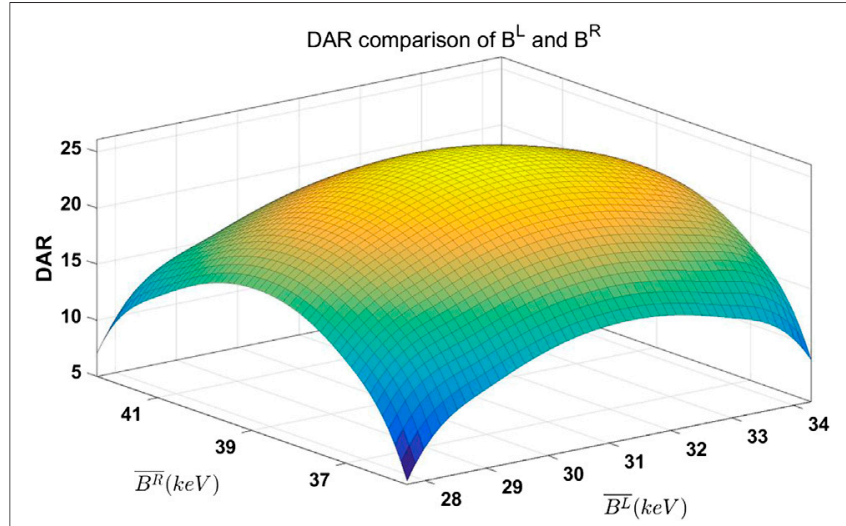

FIGURE 7 | DAR comparison of $B^{L}$ and $B^{R}$ under $10 \mathrm{keV}$ energy bin width, and $\overline{B^{L}}$ and $\overline{B^{R}}$ represent the mid-values of $B^{L}$ and $B^{R}$

energy bins, we use the multiple scanning procedure of the CT system. In our previous work, the energy resolution of the detector has been studied with several isotopes [38]. The $\mathrm{R}_{0}$ and $\mathrm{E}_{0}$ used in this article are $22.1 \%$ for $59.6 \mathrm{keV}$.

\section{Equivalent Attenuation Coefficient Curves}

In this research, the iodine mass attenuation coefficient curve was mapped to verify the availability of the model we proposed. Besides, the mass attenuation coefficient curve of gadolinium was mapped to test the accuracy of the model. The solutions with the concentration of $100 \mathrm{mg} \mathrm{I} / \mathrm{ml}$ and $100 \mathrm{mg} \mathrm{Gd} / \mathrm{ml}$ were loaded in PE centrifuge tubes with a volume of $1.5 \mathrm{ml}$. Considering the K-edge positions of iodine and gadolinium, the experimental conditions were set to $80 \mathrm{kVp}, 70 \mu \mathrm{A}$ for iodine and $90 \mathrm{kVp}$, $80 \mu \mathrm{A}$ for gadolinium.

\section{Comparison of GSSM and TASM in the Same Energy Bin Width}

The phantom used in the experiment was made of polymethyl methacrylate (PMMA) as shown in Figure 4 with 6 holes filled with iodine contrast agents with different concentrations. The inner diameter of each hole is $1 \mathrm{~mm}$. Concentrations of the iodine contrast agents are $0 \mathrm{mg} \mathrm{I} / \mathrm{ml}, 20 \mathrm{mg} \mathrm{I} / \mathrm{ml}, 25 \mathrm{mg} \mathrm{I} / \mathrm{ml}, 50 \mathrm{mg}$ $\mathrm{I} / \mathrm{ml}, 75 \mathrm{mg} \mathrm{I} / \mathrm{ml}$, and $100 \mathrm{mg} \mathrm{I} / \mathrm{ml}$ from right to left, respectively.

\section{Comparison of Different Energy Bin Widths} The $\widetilde{B^{L}}$ and $\widetilde{B^{R}}$ for different energy bin widths were used for decomposition imaging to verify the optimal width of the energy bin selected by DAR. The phantom used here was the same as that
TABLE 2 | The energy bins selected by TASM and GSSM.

\begin{tabular}{lcc}
\hline Method & Left bin (keV) & Right bin (keV) \\
\hline TASM & $23-33$ & $33-43$ \\
GSSM & $26-36$ & $34-44$
\end{tabular}

in 3.3. The energy bin widths were set to $2 \mathrm{keV}, 5 \mathrm{keV}, 8 \mathrm{keV}$, and $13 \mathrm{keV}$ for decomposition imaging.

\section{Comparative Experiment}

To illustrate the effectiveness and the superiority of GSSM compared with TASM, a comparative experiment was designed by adding a complex background to the phantom, which is made of nylon as shown in Figure 5. The nylon strips, with a thickness of $1 \mathrm{~mm}$, are used as a distraction of the iodine contrast agents in the phantom (the concentrations from right to left are $10 \mathrm{mg} \mathrm{I} / \mathrm{ml}, 15 \mathrm{mg} \mathrm{I} / \mathrm{ml}, 25 \mathrm{mg} \mathrm{I} / \mathrm{ml}, 50 \mathrm{mg}$ $\mathrm{I} / \mathrm{ml}, 75 \mathrm{mg} \mathrm{I} / \mathrm{ml}$, and $100 \mathrm{mg} \mathrm{I} / \mathrm{ml}$, respectively). The parameters of the two experiments are the same except for the energy bins.

\section{RESULTS}

\section{Equivalent Attenuation Coefficient Curves}

The measured attenuation characteristics curves of iodine and gadolinium were mapped by threshold scanning of the detector and converted to the equivalent attenuation coefficient. The comparisons with the results of GSSM are shown in Figure 6. The energy of the K-edge extreme points of iodine and gadolinium is shown in Table $\mathbf{1}$.

The results of GSSM are consistent on energy with the measured results; the errors of the extreme points are less than $3 \%$. There is a difference in $A t t_{e q}$ and this condition is more serious at lower energies. The possible reasons for this are Compton scattering and charge sharing; more additional photons at lower energies have been recorded which can result in an overestimation of photon number at low energy level.

\section{Comparison of GSSM and TASM in the Same Energy Bin Width}

The energy bin width used in this experiment was $10 \mathrm{keV}$. Corresponding DAR of $B^{L}$ and $B^{R}$ are shown in Figure 7.

The energy bins in TASM and GSSM are listed in Table 2. The decomposition images of these two methods are shown in Figure 8. We compute contrast to noise ratio (CNR) to evaluate the image quality, as shown in 


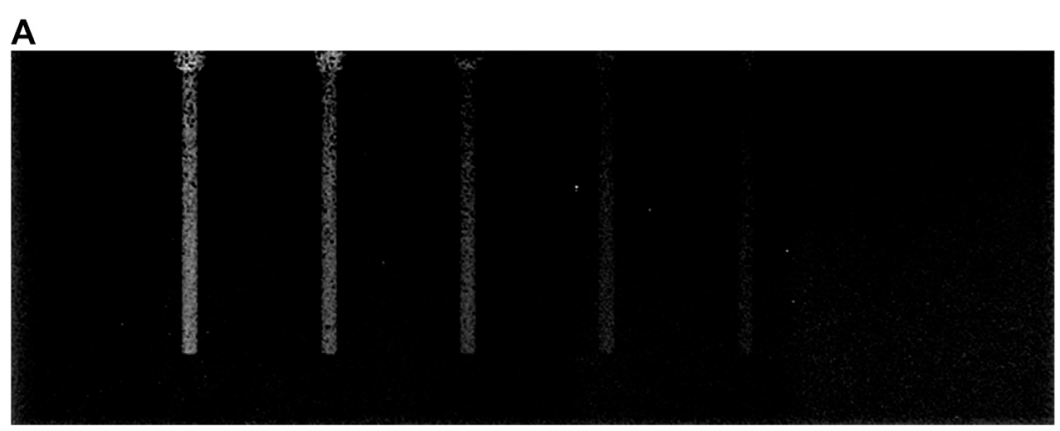

B

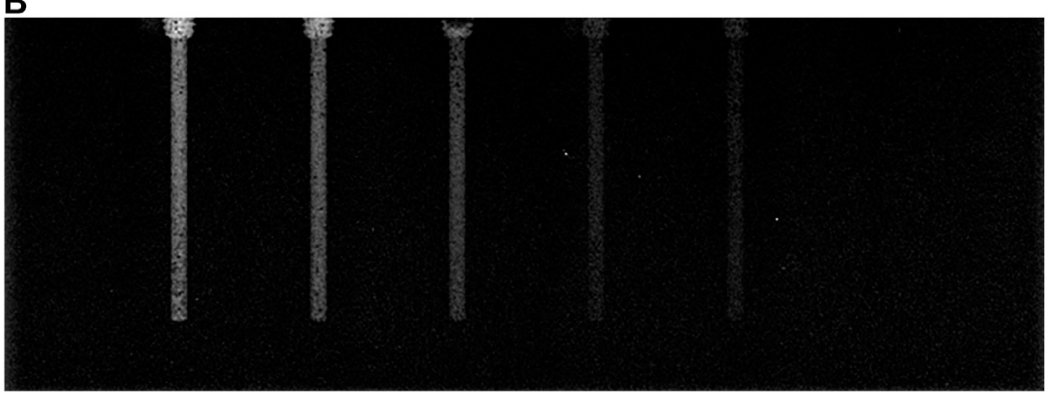

FIGURE 8 | Comparison of decomposition images obtained by TASM and GSSM: (A) decomposition image of TASM, (B) decomposition image of GSSM.

TABLE 3 | CNR comparison of decomposition images of TASM and GSSM.

\begin{tabular}{lcc}
\hline Concentration $(\mathbf{m g ~} \mathbf{~} / \mathbf{m l})$ & Method & CNR \\
\hline 20 & TASM & $0.92 \pm 0.05$ \\
25 & GSSM & $1.77 \pm 0.23$ \\
& TASM & $1.52 \pm 0.11$ \\
50 & GSSM & $2.33 \pm 0.18$ \\
& TASM & $3.29 \pm 0.32$ \\
75 & GSSM & $4.70 \pm 0.39$ \\
& TASM & $3.55 \pm 0.28$ \\
100 & GSSM & $6.16 \pm 0.54$ \\
& TASM & $4.60 \pm 0.31$ \\
& GSSM & $9.43 \pm 0.54$
\end{tabular}

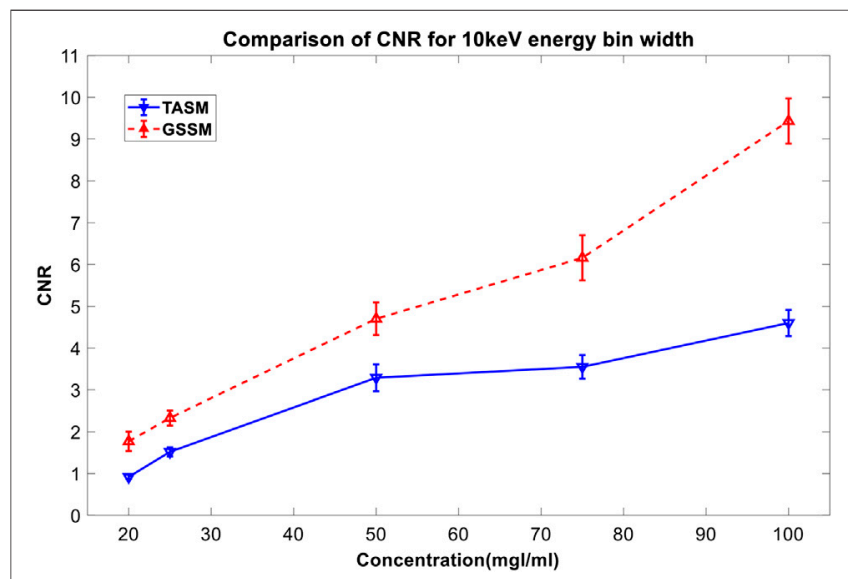

FIGURE 9 | CNR comparison for different concentrations of iodine contrast agents in Figure 8. The blue line is the CNR result of TASM; the red dashed line is the CNR result of GSSM.

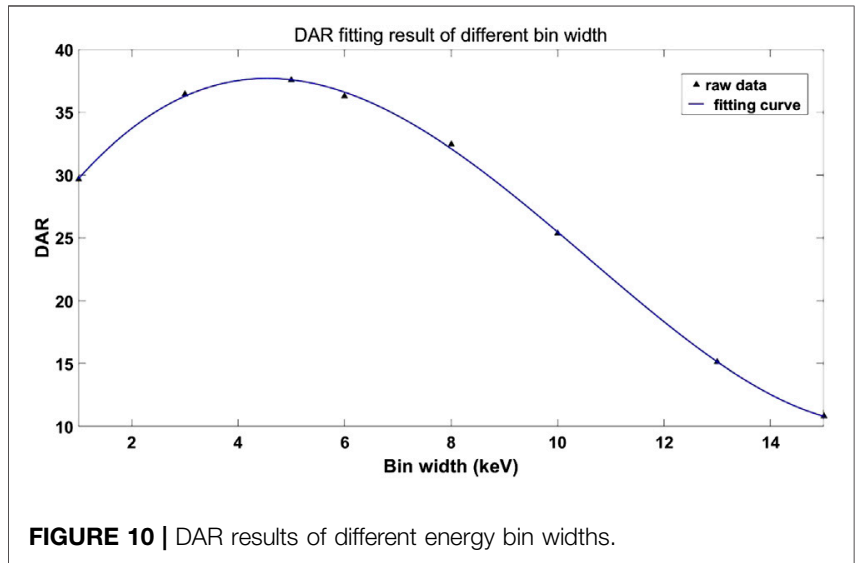

$$
C N R=\frac{\left|m_{1}-m_{2}\right|}{\sqrt{\left(\sigma_{1}^{2}+\sigma_{2}^{2}\right)}},
$$

where $m_{1}$ and $m_{2}$ are the mean values of a specific contrast agent area and the background area and $\sigma_{1}$ and $\sigma_{2}$ in the denominator indicate the standard deviation (STD) of the contrast agents area and the background, respectively. A larger CNR indicates a better image quality.

In Figure 8, both of these two decomposition images can highlight the iodine areas, but GSSM has a better performance in lower iodine concentration conditions contrast agents than TASM. The CNR values of the decomposition images are shown in Table 3 and Figure 9.

\section{Comparison of Different Energy Bin Widths}

DAR results of $\widetilde{B^{L}}$ and $\widetilde{B^{R}}$ for different energy bin widths are shown in Figure $\mathbf{1 0}$ and the optimal energy bin width is 


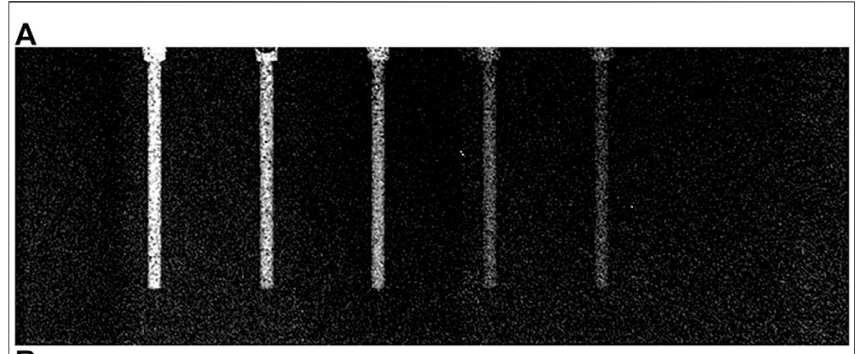

B

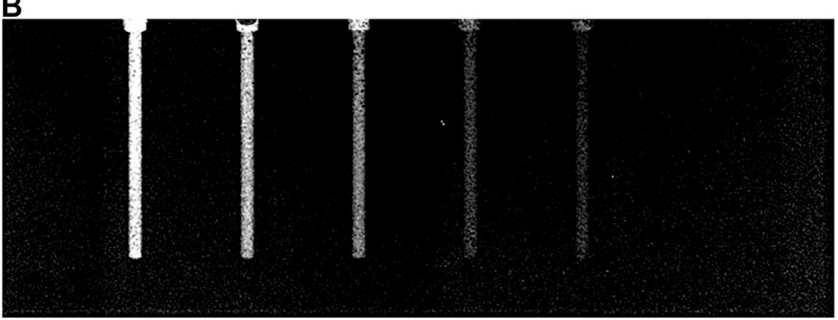

c

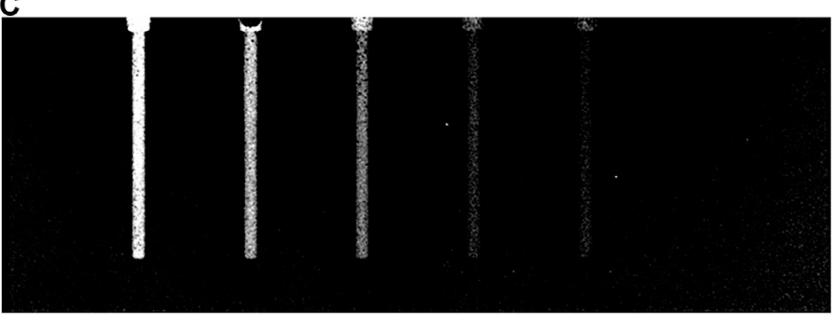

D

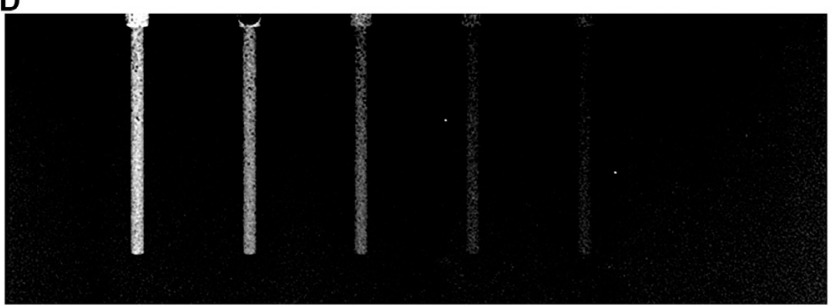

E

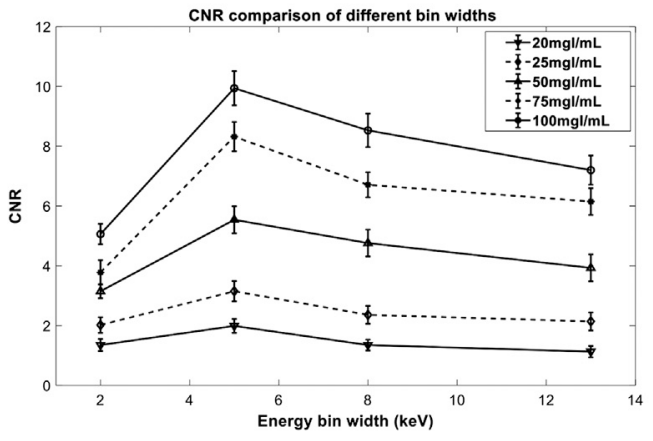

FIGURE 11 |Comparison of GSSM decomposition images with different energy bin widths: (A-D) are decomposition images of $2 \mathrm{keV}, 5 \mathrm{keV}, 8 \mathrm{keV}$, and $13 \mathrm{keV}$, respectively; (E) shows comparison of CNR values of different concentrations (100 mg l/ml, $75 \mathrm{mg} \mathrm{l} / \mathrm{ml}, 50 \mathrm{mg} \mathrm{l} / \mathrm{ml}, 25 \mathrm{mg} \mathrm{l} / \mathrm{ml}$, and $20 \mathrm{mg} \mathrm{l} / \mathrm{ml}$ from top to bottom) in the decomposition images.

determined as $5 \mathrm{keV}$. The corresponding experimental results are shown in Figure 11.

The experimental results are in good agreement with DAR results. Small energy bin width, such as $2 \mathrm{keV}$, provides good contrast of the iodine areas but strong noise in the decomposition
TABLE 4 | The energy bins used in comparison experiment.

\begin{tabular}{lcc}
\hline Method & Left bin (keV) & Right bin (keV) \\
\hline TASM & $28-33$ & $33-38$ \\
GSSM & $26-31$ & $36-41$
\end{tabular}

image (Figure 11A), while large energy bin width has a reverse effect (Figure 11D). The result shows that the $5 \mathrm{keV}$ energy bin width provides the best decomposition image quality which indicates the objective function DAR is effective.

\section{Comparative Experiment}

The energy bins for TASM and GSSM are listed in Table 4. The images are shown in Figures 12A-C, and the CNR quantitative comparison of the iodine areas in the decomposition images is shown in Figure 12D.

The normal DR image (Figure 12A) cannot distinguish the iodine areas while the images acquired by TASM and GSSM (Figures 12B,C) can exclude the interference of the background very well. Figure 12D shows that the decomposition image acquired by GSSM extracts the K-edge signal of the iodine areas better than TASM. Quantitative analysis of CNR for different concentrations of iodine solution is shown in Table 5. The CNR results are improved in GSSM compared with the TASM image; this improvement is more obvious in low concentration level because the K-edge signal drops in this condition, causing a greater deviation of energy bins and quality degradation of decomposition image. The CNR of $25 \mathrm{mg} \mathrm{I} / \mathrm{ml}$ in GSSM is equivalent to the CNR of $50 \mathrm{mg} \mathrm{I} / \mathrm{ml}$ in TASM.

\section{DISCUSSION}

This research proposes the GSSM method to optimize the extracted K-edge signal for multienergy imaging; it takes the degraded energy resolution of PCDs and the continuous x-ray spectrum into consideration. GSSM has several advantages over the traditional method. Firstly, the K-edge decomposition imaging can highlight the region of the contrast agents to get a better image quality than the ordinary DR. Secondly, GSSM is more effective than TASM in extracting the signal of K-edge, improving the quality of the decomposition images. Furthermore, GSSM can get optimized width and locations of the energy bins from the theoretical attenuation curve without the spectrum mapping process, which is more convenient for practical use. At last, due to the better performance at lower contrast agent concentration, GSSM has the potential to reduce the amount of contrast agents compared to TASM. Thus it can further improve the safety and reduce the side effect of contrast agents.

For the energy thresholds limitation of the PCD used in this study, we performed multiple scanning procedures to simulate the multienergy imaging process which can be done in a single exposure with a multithreshold PCD, such as Medipix-3 providing up to 8 energy thresholds. Besides, since the count 

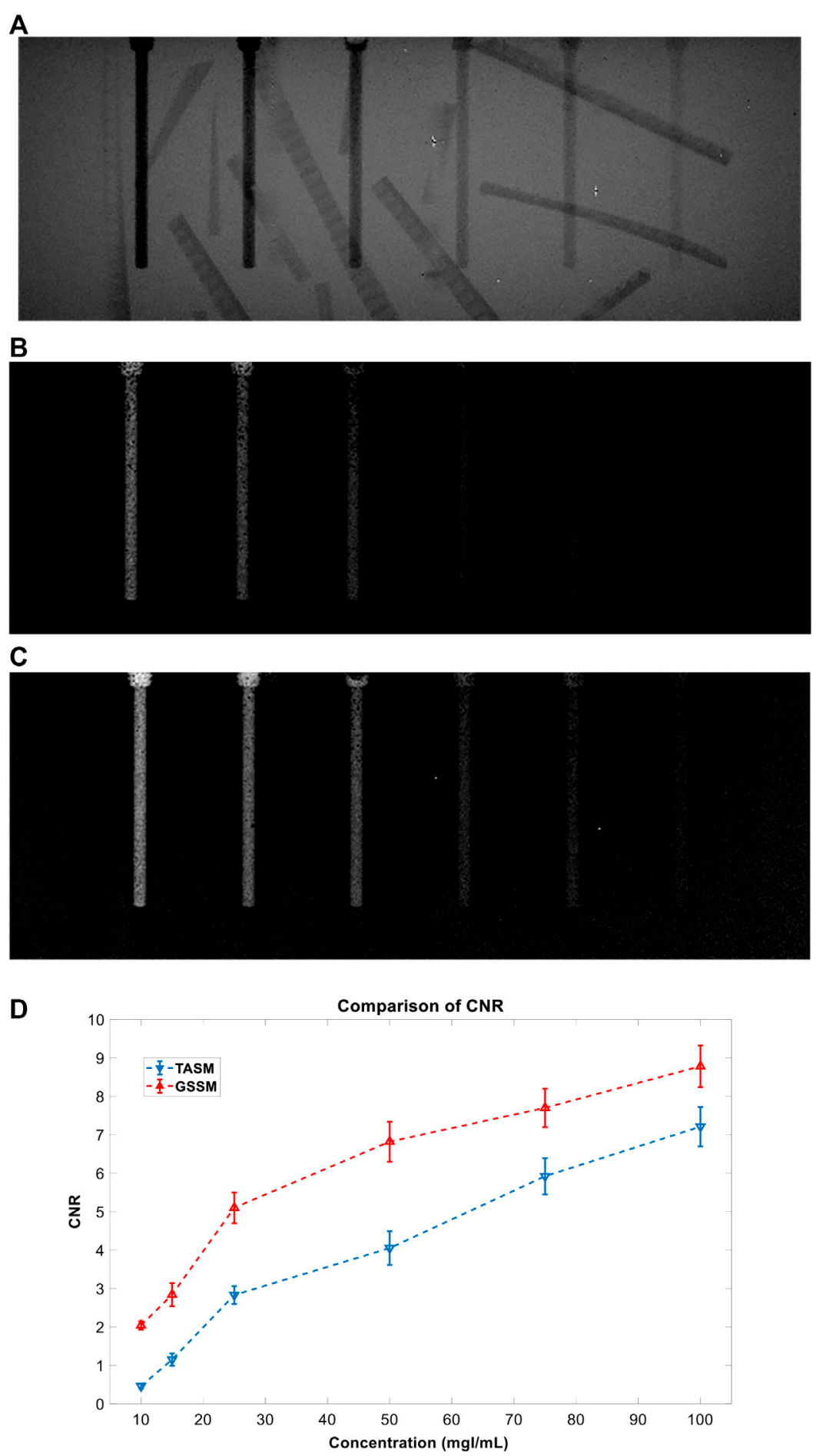

FIGURE 12 |The results of comparison experiment: (A) DR image, (B) TASM decomposition image, (C) GSSM decomposition image, and (D) CNR comparison for TASM and GSSM of the comparison experiment; the blue dashed line represents TASM CNR result and the red dashed line represents GSSM CNR result.

rate of the PCD is much lower than the conventional detector, the PCD cannot be widely used at present, but it can get exciting results in some low count rate scenarios such as the breast imaging and the small animal imaging.
It should be noted that, for the material decomposition method, there are some comprehensive models considering various effects of the imaging system [39, 40]. However, as to the determination of the energy bins in K-edge decomposition 
TABLE 5 | Quantitative analysis of CNR for different concentrations.

\begin{tabular}{lccc}
\hline $\begin{array}{l}\text { Concentration } \\
\text { (mg l/ml) }\end{array}$ & CNR_TASM & CNR_GSSM & ( $\mathbf{C}$ CNR/CNR_TASM) $\times \mathbf{1 0 0 \%}$ \\
\hline 10 & & & \\
15 & $0.46 \pm 0.06$ & $2.04 \pm 0.11$ & $343.48 \%$ \\
25 & $1.15 \pm 0.16$ & $2.84 \pm 0.30$ & $146.96 \%$ \\
50 & $2.83 \pm 0.23$ & $5.10 \pm 0.40$ & $80.21 \%$ \\
75 & $4.05 \pm 0.44$ & $6.82 \pm 0.52$ & $68.40 \%$ \\
100 & $5.92 \pm 0.47$ & $7.70 \pm 0.50$ & $30.07 \%$ \\
& $7.21 \pm 0.51$ & $8.78 \pm 0.54$ & $21.78 \%$ \\
\hline
\end{tabular}

imaging, a Gaussian model can get better results than the current methods; a more accurate result can be expected if the model can consider other effects of the imaging system. Besides, a difference between the heights of equivalent attenuation coefficient curves is noticed in Imaging System and its possible explanations are factors like Compton scattering and charge sharing. Therefore, specially designed correction methods could be helpful to achieve better performance. Moreover, the energy bin widths on both sides of the K-edge are equal in this preliminary result. Future study can put attention on imaging with nonequal energy bin widths.

\section{CONCLUSION}

The image contrast can be improved by using the K-edge imaging technique. This research proposes a more convenient and efficient energy bins determination method, GSSM, for extracting the K-edge signal in multienergy imaging based on the PCDs. The decomposition image acquired by GSSM has better quality than that acquired by TASM in terms of CNR. Because the improvement is especially considerable under low contrast agent concentration situation, our method has a potential to reduce the

\section{REFERENCES}

1. Lewin JM, Isaacs PK, Vance V, and Larke FJ. Dual-energy contrast-enhanced digital subtraction mammography: feasibility. Radiology (2003) 229:261-8. doi:10.1148/radiol.2291021276

2. Si-Mohamed S, Thivolet A, Bonnot PE, Bar-Ness D, Képénékian V, Cormode $\mathrm{DP}$, et al. Improved peritoneal cavity and abdominal organ imaging using a biphasic contrast agent protocol and spectral photon counting computed tomography K-edge imaging. Invest Radiol (2018) 53 (10):629-39.doi:10. 1097/RLI.0000000000000483

3. Zhang L, Zhou C, Schoepf U, Sheng H, Wu S, Krazinski A, et al. Dual-energy CT lung ventilation/perfusion imaging for diagnosing pulmonary embolism. Eur Radiol (2013) 23 (10):2666-75. doi:10.1007/s00330-013-2907-x

4. Badea C, Johnston S, Qi Y, Ghaghada K, and Johnson G. Dual-energy micro-CT imaging for differentiation of iodine- and gold-based nanoparticles. Proc SPIE (2011) 7961:79611X. doi:10.1117/12.878043

5. Achenbach S, Anders K, and Kalender WA. Dual-source cardiac computed tomography: image quality and dose considerations. Eur Radiol (2008) 18: 1188-98. doi:10.1007/s00330-008-0883-3

6. Panetta D. Advances in X-ray detectors for clinical and preclinical Computed Tomography[J]. Nucl Instrum Methods Phys Res Sect A Accel Spectrom Detect Assoc Equip (2016) 809: 2-12. doi:10.1016/j.nima.2015.10.034 requirement of contrast agent in multienergy imaging, which could be valuable in the clinical applications.

\section{DATA AVAILABILITY STATEMENT}

The raw data supporting the conclusions of this article will be made available by the authors, without undue reservation.

\section{AUTHOR CONTRIBUTIONS}

$\mathrm{ZZ}$ was responsible for the method and experimental design as well as the writing of the article, JH and XZ assisted in the experiment and data processing, QX and ML provided us with software and algorithm help, and LW, CW, and ZW gave full guidance and help in the design and completion of the research.

\section{FUNDING}

This work is supported by the National Key R\&D Program of China (Grant No. 2016YFC0100400), the Science and Technology Service Network Initiative of Chinese Academy of Sciences (Grant No. KFJ-STS-QYZD-193), the Key Technology Research and Development Team Project of Chinese Academy of Sciences (Grant No. GJJSTD20200004), and the National Natural Science Foundation of China (Grant No.11975250).

\section{ACKNOWLEDGMENTS}

We are very grateful to Shu Yanfeng for his help in the design and production of the phantom.

7. Maturen KE, Kleaveland PA, Kaza RK, Liu PS, Quint LE, Khalatbari SH, et al Aortic endograft surveillance: use of fast-switch $\mathrm{kVp}$ dual-energy computed tomography with virtual noncontrast imaging. J Comput Assist Tomogr (2011) 35: 742-6. doi:10.1097/RCT.0b013e3182372c14

8. Goo HW, and Goo JM. Dual-energy CT: new horizon in medical imaging. Korean J Radiol (2017) 18:555-69. doi:10.3348/kjr.2017.18.4.555

9. Wang X, Meier D, Taguchi K, Wagenaar DJ, Patt BE, and Frey EC. Material separation in X-ray CT with energy resolved photon-counting detectors. Med Phys (2011) 38 (3):1534-46. doi:10.1118/1.3553401

10. Schlomka JP, Roessl E, Dorscheid R, Dill S, Martens G, Istel T, et al. Experimental feasibility of multi-energy photon-counting K-edge imaging in pre-clinical computed tomography. Phys Med Biol (2008) 53 (15): 4031-47. doi:10.1088/0031-9155/53/15/002

11. Taguchi K, and Iwanczyk JS. Vision 20/20: single photon counting $\mathrm{x}$-ray detectors in medical imaging. Med Phys (2013) 40(10):100901. doi:10.1118/1. 4820371

12. Schmitzberger FF, Fallenberg EM, Lawaczeck R, Hemmendorff M, Moa E, Danielsson $\mathrm{M}$, et al. Development of low-dose photon-counting contrastenhanced tomosynthesis with spectral imaging. Radiology (2011) 259:558-64. doi:10.1148/radiol.11101682

13. Roessl E, and Proksa R, K-edge imaging in x-ray computed tomography using multi-bin photon counting detectors. Phys Med Biol (2007) 52:4679-96. doi:10.1088/0031-9155/52/15/020 
14. Shikhaliev PM. Photon counting spectral CT: improved material decomposition with K-edge-filtered x-rays. Phys Med Biol (2012) 57(6): 1595. doi:10.1088/0031-9155/57/6/1595

15. Cunningham IA, Westmore MS, and Fenster A. A spatial-frequency dependent quantum accounting diagram and detective quantum efficiency model of signal and noise propagation in cascaded imaging systems. Med Phys (1994) 21:417-27. doi:10.1118/1.597401

16. Anderson NG, Butler AP, Scott NJ, Cook NJ, Butzer JS, Schleich N, et al. Spectroscopic (multi-energy) CT distinguishes iodine and barium contrast material in MICE. Eur Radiol (2010) 20:2126-34. doi:10.1007/s00330-010-1768-9

17. Liang L, Li R, Zhang S, and Chen Z. Simultaneous X-ray fluorescence and K-edge CT imaging with photo-counting detectors. Proc SPIE (2016) 9967: 99670F. doi:10.1117/12.2238528

18. Ge Y, Zhang R, Li K, and Chen GH. K-edge energy-based calibration method for photon counting detectors. Phys Med Biol (2018) 63:015022. doi:10.1088/ 1361-6560/aa9637

19. Sossin A, Rebuffel V, Tabary J, Létang JM, Freud N, Verger L, et al. "Influence of scattering on material quantification using multi-energy $x$-ray imaging," in 2014 IEEE Nuclear Science Symposium and Medical Imaging Conference (NSS/MIC), Seattle, WA, November 8-15, 2014 (IEEE) (2014).

20. Kim JC, Anderson SE, Kaye W, Zhang F, Zhu Y, Kaye SJ, et al. Charge sharing in common-grid pixelated CdZnTe detectors. Nucl Instrum Methods Phys Res (2010) 654 (1):233-43. doi:10.1016/j.nima.2011.06.038

21. Wang AS, Harrison D, Lobastov V, and Tkaczyk JE. Pulse pileup statistics for energy discriminating photon counting x-ray detectors. Med Phys (2011) 38: 4265-75. doi:10.1118/1.3592932

22. Shikhaliev PM, Fritz SG, and Chapman JW. Photon counting multienergy $\mathrm{x}$-ray imaging: effect of the characteristic $\mathrm{x}$ rays on detector performance. Med Phys (2009) 36:5107. doi:10.1118/1.3245875

23. Taguchi K, Frey EC, Wang X, Iwanczyk JS, and Barber WC. An analytical model of the effects of pulse pileup on the energy spectrum recorded by energy resolved photon counting x-ray detectors. Med Phys (2010) 37:3957-69. doi:10.1118/1.3429056

24. Lee Y, Lee AC, and Kim H. A Monte Carlo simulation study of an improved K-edge log-subtraction X-ray imaging using a photon counting CdTe detector. Nucl Instrum Methods Phys Res (2016) 830:381-90. doi:10.1016/j.nima.2016.06.024

25. Pani S, Saifuddin S, Ferreira FIM, Henthorn N, Seller P, Sellin PJ, et al. High energy resolution hyperspectral X-ray imaging for low-dose contrastenhanced digital mammography. IEEE Trans Med Imaging (2017) 36: 1784-95. doi:10.1109/TMI.2017.2706065

26. Pani S, Saifuddin S, Ferreira F, Scuffham J, Stratmann P, Wilson M, et al. "Optimization of K-edge subtraction imaging using a pixellated spectroscopic detector," in Nuclear Science Symposium and Medical imaging Conference, Anaheim, CA, October 3, 2012 (IEEE), 3063-6 (2012)

27. Feng M, Xu J, Treb K, Zhang R, Chen G-H, and Li K. Spectrum optimization in photon counting detector based iodine K-edge CT imaging. Proc Medical imaging 2019: Physics of Medical imaging (2019) 10948:109481C. doi:10.1117/12.2512893

28. Ding H, Molloi S. Image-based spectral distortion correction for photoncounting X-ray detectors. Med Phys (2012) 39:1864-76. doi:10.1118/1.3693056

29. He P, Wei B, Cong W, and Wang G. Optimization of K-edge imaging with spectral CT. Med Phys (2012) 39:6572. doi:10.1118/1.4754587
30. Meng B, Cong W, Xi Y, De Man B, and Wang G. Energy window optimization for X-ray K-edge tomographic imaging. IEEE Trans Biomed Eng (2016) 63: 1623-30. doi:10.1109/TBME.2015.2413816

31. Lee SW, Choi YN, Cho HM, Lee YJ, Ryu HJ, and Kim HJ. A Monte Carlo simulation study of the effect of energy windows in computed tomography images based on an energy-resolved photon counting detector. Phys Med Biol (2012) 57:4931-49. doi:10.1088/0031-9155/57/15/4931

32. Roessl E, Brendel B, Engel KJ, Schlomka JP, Thran A, and Proksa R, Sensitivity of photon-counting based K-edge imaging in X-ray computed tomography. IEEE Trans Med Imaging (2011) 30 (9):1678-90. doi:10.1109/TMI.2011.2142188

33. Hubbell JH, Seltzer SM. Tables of x-ray mass attenuation coefficients and mass-energy absorption coefficients $1 \mathrm{keV}$ to $20 \mathrm{meV}$ for elements $\mathrm{z}=1$ to 92 and 48 additional substances of dosimetric interest. Gaithersburg, MD: National Institute of Standards and Technology (1995). Available from: http://physics.nist.gov/pml/data/xraycoef/index.cfm.

34. Poludniowski G, Landry G, DeBlois F, Evans PM, and Verhaegen F. SpekCalc: a program to calculate photon spectra from tungsten anode $\mathrm{x}$-ray tubes. Phys Med Biol (2009) 54(19): N433-8. doi:10.1088/0031-9155/54/19/N01

35. Chen SC, Jong WL, Harun AZ Evaluation of X-ray beam quality based on measurements and estimations using SpekCalc and Ipem78 models. Malays J Med Sci (2012) 19(3):22-8.

36. Kalluri KS, Mahd M, and Glick SJ. Investigation of energy weighting using an energy discriminating photon counting detector for breast CT. Med Phys (2013) 40:081923. doi:10.1118/1.4813901

37. Cheng Z, Li M, Xu Q, Zhang Z, Hu J, Wei C, et al. Improved projection-based energy weighting for spectral CT. Radiation Detection Technology and Methods (2019) 3:28. doi:10.1007/s41605-019-0106-2

38. Li ZH, Yun MK, Jiang XP, Zhang ZD, Cheng ZW, Wei CF, et al. Energy calibration method of the photon counting detector based on continuous $\mathrm{x}$-ray spectrum[J]. CT Theory and Applications (2018) 27(3):363-72. doi:10.15953/j. 1004-4140.2018.27.03.09

39. Ehn S, Sellerer T, Mechlem K, Fehringer A, Epple M, Herzen J, et al. Basis material decomposition in spectral CT using a semi-empirical, polychromatic adaption of the Beer-Lambert model [J]. Phys Med Biol (2017) 62 (1):N1-17. doi:10.1088/1361-6560/aa4e5c

40. Cai C, Rodet T, Legoupil S, and Mohammad-Djafari A. A full-spectral Bayesian reconstruction approach based on the material decomposition model applied in dual-energy computed tomography. Med Phys (2013) 40 (11):111916. doi:10.1118/1.4820478

Conflict of Interest: The authors declare that the research was conducted in the absence of any commercial or financial relationships that could be construed as a potential conflict of interest.

Copyright (C) 2021 Zhang, Zhang, Hu, Xu, Li, Wei, Wei and Wang. This is an openaccess article distributed under the terms of the Creative Commons Attribution License (CC BY). The use, distribution or reproduction in other forums is permitted, provided the original author(s) and the copyright owner(s) are credited and that the original publication in this journal is cited, in accordance with accepted academic practice. No use, distribution or reproduction is permitted which does not comply with these terms. 


\section{APPENDIX 1}

\section{The Derivation of DAR}

This research establishes the objective function, difference of attenuation to relative standard deviation ratio (DAR), model to optimize the energy bin selection. The DAR definition is shown in

$$
D A R=\frac{\Delta A t t_{e q}}{N}
$$

where $\Delta A t t_{e q}$ represents the difference of equivalent attenuation coefficient between the left and the right energy bins beside the $\mathrm{K}$-edge. $\mathrm{N}$ represents the total noise level of these two images. The quality of the decomposition image is the best when the DAR is maximized.

The attenuation characteristic $\mathrm{A}$ of the acquired image can be expressed as follows:

$$
A=-\ln \frac{S}{S_{0}}=-\ln \frac{\left(\int_{B} P_{G}(E) d E\right) e^{-A t t_{e q}(B)(\rho d)}+N_{R}}{\int_{B} P_{G}(E) d E}
$$

where $\mathrm{N}_{\mathrm{R}}$ is the random noise component and $\operatorname{Att}_{\text {eq }}(B)$ is the equivalent attenuation coefficient.

The mean value and variance of $A$ are calculated in

$$
\begin{gathered}
E(A)=A t t_{e q}(B)(\rho d), \\
\operatorname{Var}(A)=\frac{1}{\left(\int_{B} P_{G}(E) d E\right) \mathrm{e}^{-A t t_{e q}(B)}(\rho d)}
\end{gathered}
$$

Relative standard deviation is used as the representation of the image's noise level, and then $\mathrm{N}$ is expressed as follows:

$$
N=\sqrt{\frac{1}{\left(\operatorname{Att}_{e q}(B)^{\star}(\rho \mathrm{d})\right)^{2 *}\left(\int_{\mathrm{B}} \mathrm{P}_{\mathrm{G}}(\mathrm{E}) \mathrm{dE}\right) \mathrm{e}^{-\mathrm{Atteq}_{e q}(\mathrm{~B})(\rho \mathrm{d})}}}
$$

The corresponding representation of the left and the right energy bins can be expressed as in (5) and (6).

For the left bin of K-edge:

$$
A^{L}=A t t_{e q}\left(B^{L}\right)(\rho d)-\frac{N_{R}^{L}}{\left(\int_{B^{L}} P_{G}(E) d E\right) \mathrm{e}^{-A t t_{e q}\left(B^{L}\right)(\rho d)}}
$$

$$
\begin{aligned}
E\left(A^{L}\right) & =A t t_{e q}\left(B^{L}\right)(\rho \mathrm{d}) \\
\operatorname{Var}\left(A^{L}\right) & =\frac{1}{\left(\int_{B^{L}} N_{S}(E) d E\right) \mathrm{e}^{-A t t t_{e q}\left(B^{L}\right)(\rho d)}}
\end{aligned}
$$

For the right bin of K-edge:

$$
\begin{aligned}
A^{R} & =\operatorname{Att}_{e q}\left(B^{R}\right)(\rho \mathrm{d})-\frac{N_{R}^{R}}{\left(\int_{B^{R}} P_{G}(E) d E\right) e^{-A t t_{e q}\left(B^{R}\right)(\rho d)}} \\
E\left(A^{R}\right) & =\operatorname{Att}_{e q}\left(B^{R}\right)(\rho \mathrm{d}) \\
\operatorname{Var}\left(A^{R}\right) & =\frac{1}{\left(\int_{B^{R}} P_{G}(E) d E\right) \mathrm{e}^{-A t t t_{e q}\left(B^{R}\right)(\rho d)}}
\end{aligned}
$$

The $\Delta A t t_{e q}$ and $\mathrm{N}$ in DAR can be expressed as follows:

$$
\begin{aligned}
& \Delta A t t_{e q}=A^{R}-A^{L}=E\left(A^{R}\right)-E\left(A^{L}\right) \\
& =\operatorname{Att}_{e q}\left(B^{R}\right)(\rho \mathrm{d})-\operatorname{Att}_{e q}\left(B^{L}\right)(\rho \mathrm{d}) \\
& N=\frac{\sqrt{\operatorname{Var}\left(A^{L}\right)+\operatorname{Var}\left(A^{R}\right)}}{A t t_{e q}\left(B^{R}\right)(\rho d)-A t t_{e q}\left(B^{L}\right)(\rho d)} \\
& =\frac{\sqrt{\left(\int_{B^{L}} P_{G}(E) d E\right) \mathrm{e}^{-A t t_{e q}\left(B^{L}\right)(\rho d)}+\frac{1}{\left(\int_{B^{R}} P_{G}(E) d E\right) \mathrm{e}^{-A t t_{e q}\left(B^{R}\right)(\rho d)}}}}{A t t_{e q}\left(B^{R}\right)(\rho d)-A t t_{e q}\left(B^{L}\right)(\rho d)}
\end{aligned}
$$

The final DAR is shown in

$$
\operatorname{DAR}\left(B^{L}, B^{R}\right)=\frac{\left(\operatorname{Att}_{e q}\left(B^{R}\right) *(\rho d)-\operatorname{Att}_{e q}\left(B^{L}\right) *(\rho d)\right)^{2}}{\sqrt{\left(\int_{B^{L}} P_{G}(E) d E\right) \mathrm{e}^{-A t t_{e q}\left(B^{L}\right) *(\rho d)}}+\frac{1}{\left(\int_{B^{R}} P_{G}(E) d E\right) \mathrm{e}^{-A t e q}\left(B^{R}\right)^{*(\rho d)}}}
$$

where ( $\rho d)$ is the mass thickness of the sample, $B^{L}$ and $B^{R}$ are the left and the right energy bins beside the K-edge, and $\operatorname{Att}_{e q}\left(B^{\mathrm{L}}\right)$ and $\operatorname{Att}_{e q}\left(B^{\mathrm{R}}\right)$ are the equivalent attenuation coefficients of $B^{\mathrm{L}}$ and $\mathrm{B}^{\mathrm{R}}$. 